\title{
Sizes of Confirmed NGC 5128 Globular Clusters
}

\author{
Doug Geisler ${ }^{1}$, M. Gómez ${ }^{1}$ \\ K. A. Woodley ${ }^{2}$, W. E. Harris ${ }^{2}$ \\ and G. L. H. Harris ${ }^{3}$ \\ ${ }^{1}$ Departamento de Fisica, Universidad de Concepción, Concepción, Chile \\ email: dgeisler@astro-udec.cl \\ ${ }^{2}$ Department of Astronomy, McMaster University, Toronto, Ontario, Canada \\ ${ }^{3}$ Department of Astronomy, University of Waterloo, Toronto, Ontario, Canada
}

\begin{abstract}
We present results from a new wide-field study of the NGC 5128 globular cluster system. We have obtained new high resolution images with the Magellan 6.4m + IMACS camera. Our images cover an area of $1.2 \times 1.2$ sq. degrees and have a seeing of 0.45 ". This allows us to not only resolve most of the globular clusters (GCs) but also derive their structural parameters. These are combined with existing Washington photometry in order to select by metallicity. We present here results for a subsample of 359 GCs which includes all currently confirmed GC members of the system. Our derived sizes are in very good agreement with those derived from ACS data. We find, as expected, that the metal-rich GCs in the inner regions $\left(\mathrm{r}<10^{\prime}\right)$ are $26 \%$ smaller than their metal-poor components, but in the outer region this normal trend is reversed. We compare our GCs to previous results for GCs, UCDs, etc. in the luminosity - size plane and find substantial overlap between different types of objects, indicating more of a continuum in these properties.
\end{abstract}

Keywords. galaxies: star clusters

\section{Introduction}

At a distance of $\sim 3.5 \mathrm{Mpc}$, NGC 5128 is the nearest giant elliptical. It contains several thousand globular clusters (GCs). They are ideal targets for addressing a number of important questions regarding $\mathrm{GC}$ and galaxy formation.

\section{Observations and Analysis}

We have recently obtained superb new imaging data for this system with the Magellan $6.5 \mathrm{~m}+$ IMACS. Our images cover an area of $1.2 \times 1.2$ sq. degrees in a set of 25 fields $15.4^{\prime}$ on a side. Pixels are 0.11 " and the seeing was 0.45 ". This allows us to cover essentially the entire GC system.

This data allows us to not only resolve most of the GCs but also derive their structural parameters, using the ISHAPE program from S. Larsen. We have first applied this technique to a total of 359 objects which includes ALL currently confirmed GC members from radial velocity data.

The sizes we derive show excellent agreement with those found from HST/ACS data using an entirely independent technique. This gives us increased confidence in our sizes. We then combine our structural parameters with our existing Washington photometry which provides an excellent metallicity indicator. 


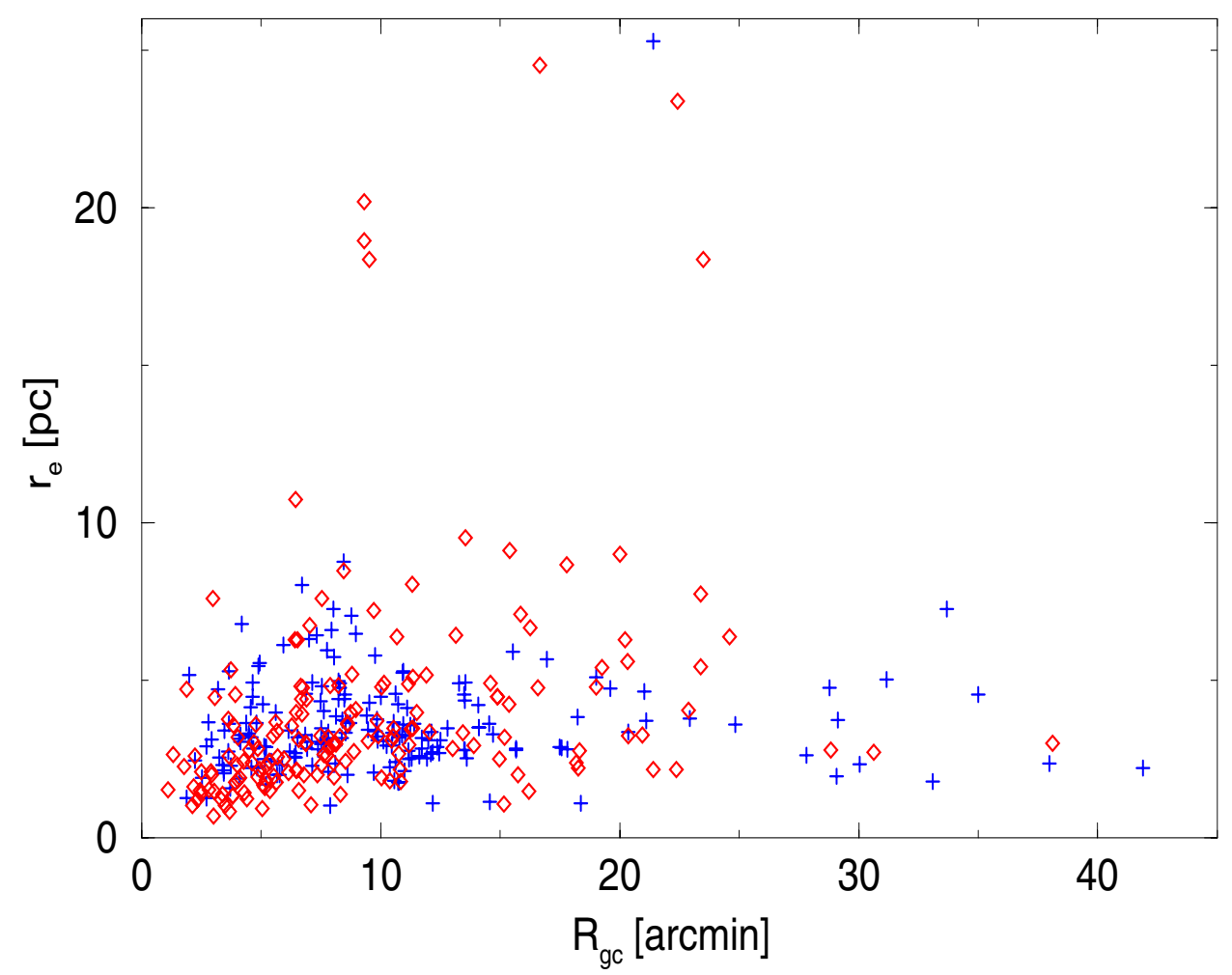

Figure 1. GC size (in pc) vs. galactocentric distance (in ') for 359 confirmed GCs in NGC 5128. (Red) squares are GCs more metal-rich than $[\mathrm{Fe} / \mathrm{H}]=-1$ and (blue) crosses are GCs more metal-poor than this limit.

\section{Results}

We divide our sample into metal-rich (RGCs) and metal-poor (BGCs) clusters and plot their sizes vs. galactocentric radius in Fig. 1. In the inner 10', we find the usual result: the 107 BGCs are $26 \%$ larger than the 107 RGCs. BUT in the outer region, the 79 BGCs are 23\% SMALLER than the 58 RGCs! Note the substantial fraction of RGCs with sizes $>8 \mathrm{pc}$ in this region. This is the first time such a clear trend has been found. Perhaps projection effects are responsible for this difference.

We finally compare our clusters to previous results for star clusters, UCDs, etc . in the $M_{V}$ :size plane. Although one speaks of different types of objects, implying different behavior in such a diagram, we instead find substantial overlap between different types of objects, indicating more of a continuum in these properties.

\section{Acknowledgements}

D.G. gratefully acknowledges support from the Chilean Centro de Astrofísica FONDAP No. 15010003. 